\title{
Multi-dimensional Interactive City Exploration through Mixed Reality
}

\author{
Iris Herbst, Anne-Kathrin Braun, Rod McCall, Wolfgang Broll \\ Collaborative Virtual and Augmented Environments Department, Fraunhofer FIT
}

Sankt Augustin, Germany

\begin{abstract}
In this paper we present a pervasive outdoor mixed reality edutainment game for exploring the history of a city in the spatial and the temporal dimension, which will closely couple the real environment with the virtual content. The game provides a new and unique user experience, which links rich interactive content to time and places. We introduce the development of such a game, including a universal mechanism to define and setup multi-modal user interfaces for game challenges.
\end{abstract}

KEYWORDS: Augmented Reality (AR), Multimodal Interfaces, Pervasive Gaming, Mixed Reality (MR), Presence.

INDEX TERMS: H.5.1 [Multimedia Information Systems]: Artificial, augmented, and virtual realities - Audio input/output H.5.2 [User Interfaces]: Input devices and strategies. I.3.1 [Hardware Architecture]: Input devices. I.3.6 [Computer Graphics]: Methodology and Techniques - Interaction Techniques. K.8.0 [Personal Computing]: General - Games.

\section{INTRODUCTION}

Pervasive games and mobile edutainment applications represent a major advance for game players and developers. Such games use information and communication technology (ICT) and AR technology to overcome the boundaries of traditional gaming environments by creating new, extended ones, where the real environment of the user becomes an intrinsic component of the overall game.

We developed a system that allows for a 4D multimedia experience. Whereas former projects do not provide a sense of time but only display the historic scenes to the spectator, we provide interfaces and interaction techniques allowing the player to interact with the city and to experience its temporal changes. Instead of static, timeline-based game play, we provide a nonlinear experience of the individual historic time periods. This impression is enforced by rich interactive content placed carefully in certain locations.

\section{THE TIMEWARP APPROACH}

TimeWarp creates an environment where the real and virtual elements combine to create a unified experience in which the user feels present, thus it is the relationship between the real and the virtual which is key. We chose initially to base our work two classic types of presence: social (the feeling of being with others) and physical presence (being in the mediated environment) [1].

The game is staged in the old part of Cologne, within an area of about 1.5 square miles. The story is about the Heinzelmännchen of Cologne, which were small elves, helping the citizens of Cologne during the night, until they suddenly disappeared. We extend this legend by spreading the rumor that the elves fell into time holes.

IEEE Virtual Reality 2008

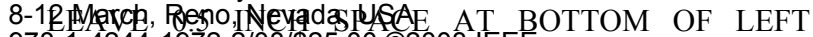

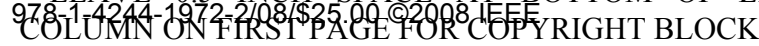

Thus, they are still in the city, but they are captured in different time periods. They goal of the game is to find the Heinzelmännchen within the individual epochs (Figure 1) by the means of time travel and bring them back into the present, by solving special challenges. Therefore, each player is equipped with a mobile AR system and a handheld-based information device, which enables her to see the elves and to visit different time periods - roman, medieval, new age and even the future.

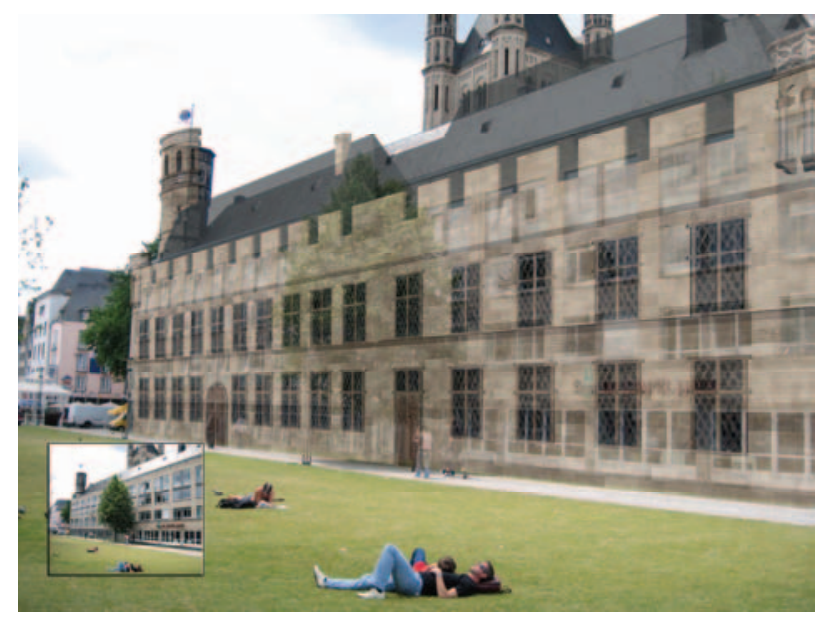

Figure 1. Virtual reconstruction of a historic building (referring to medieval time) compared to real world setting (small image)

\section{System ConcePt}

The TimeWarp AR application is based on our AR/VR framework MORGAN [3] and uses our AR/VR viewer Marvin for $3 \mathrm{D}$ visualization.

Each player is equipped with two systems: a laptop-based mobile AR system which augments the real environment, and a PDA-based information device. The latter provides several services supporting the player during the game, e.g. an interactive map showing the current position of the player in satellite or map mode (similar to services such as Google maps) allows for easy player guidance within the city. The devices communicate via a Bluetooth connection.

We use a head-worn optical see-through display and a GPS receiver for positioning and an inertial tracker sensor for orientation tracking attached to the display. The total weight is less than $4.4 \mathrm{lbs}$. Interaction devices are either a standard Bluetooth mouse or a gyroscopic mouse.

\section{USER INTERFACE DESIGN AND INTERACTION}

\subsection{User Controls}

Being in public, uncontrolled and unrestricted space sets up limitations to the possible user interaction due to the fact that the 
hardware to realize user control has to be carried by the user. Furthermore user control should contribute to the game experience. Consequentially, controller functionalities were restricted to a core set, avoiding 2D control widgets such as menus or sliders, taking advantage of prior design experiences with other games and game-like applications.

\section{Physical Proximity}

The first core interaction control is realized through proximity in the real world. Being location-aware, the system reacts on the proximity of the player to a location: in our approach, we have three proximity ranges: outside, near, and at a game location. A sound icon additionally informs the player when they are close to a game location. To avoid unintentional use of the AR interaction device, input is only enabled while the player is at a game location. A specific sound icon informs the player about the activation and deactivation. Additionally, particular actions may be executed immediately.

An example is a virtual character which starts talking depending on a player's proximity.

Focus, action and feedback

The second core interaction control is based on focus and click events. Focus either is controlled with a gaze-based view pointer, a view related crosshair, or by stepping through selectable items in predefined order using the mouse wheel. The view pointer is controlled by the viewing position and orientation, thus the view pointer makes advantage of the user tracking which is anyway required for registration. If a game item is in focus, in this first version of the game, its bounding box is drawn around it.

Examples for actions and feedback associated with a click are items appearing on the PDA interface, items disappearing in the MR augmentation, or sound icons associated to a click.

\section{Placing a virtual item}

The third core interaction control was inspired by the Wii remote controller. Movements with a gyroscopic mouse in mid air control the orientation of an item, whereas translation speed is controlled by the mouse wheel. Actions and feedback associated with a click can be manifold as well (see above), depending on the individual needs of the designed challenge.

\section{Addressing technical issues}

Test runs with an early prototype have shown that the user needs some kind of feedback that the system is still working properly even if she is not at a game location. These needs arise from the sparse virtual content and inaccurate tracking. We address this issue by providing visual feedback: a graphical element displayed in the HMD represents the reception of GPS position updates. Another issue is the pretty low accuracy of GPS: virtual content is floating around rather than stable aligned with the real world. In our first prototype, the players may freeze their position avoiding floating virtual elements. This however, is rather considered a work-around than a final solution. In our future versions we plan to incorporate markerless computer vision-based tracking mechanisms for this purpose.

\subsection{Spatial and Temporal Augmentation}

The traditional laboratory based view of presence may not be appropriate for real life situations such as those experienced with MR. Therefore we use the term spatial and temporal augmentation to refer to multimedia content that is embedded in spatial and temporal dimension of the environment. In our approach spatial augmentation is extended to all kinds of mobile devices: content provided on the mobile AR system or the hand- held system is location-depending. Temporal augmentation refers to the user's experience of being in a certain time period. Visiting the same place in different time periods allows witnessing its change over time (Figure 2); the game concept of time travel through time portals instead of a timeline-based widget control for selecting the current time period additionally contributes to this experience. Furthermore temporal changes and augmentations are reflected across the range of devices.
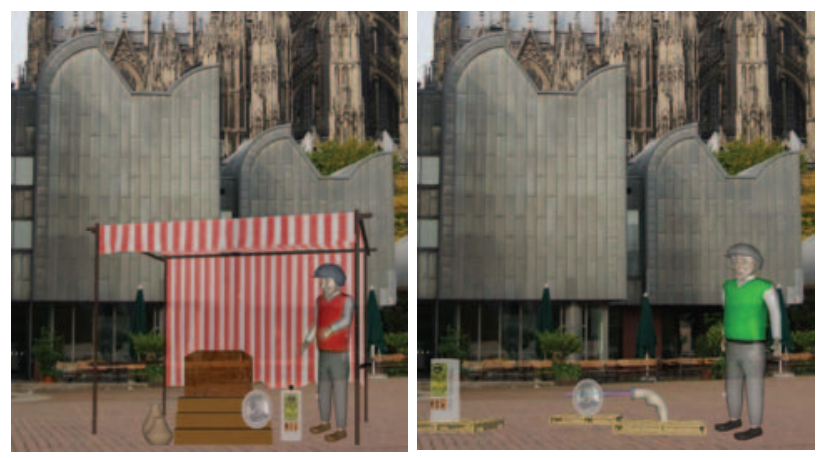

Figure 2. A market place displayed in different time periods (medieval - left, roman - right)

\section{CONCLUSION AND FUTURE WORK}

We presented TimeWarp - a novel pervasive augmented reality game allowing for experiencing a city through time and space. We introduced how mobile AR technology in combination with non linear game play can be used to achieve various kinds of presence, including spatial, physical, social, and temporal presence.

In our future work we intend to significantly enhance the registration of the virtual objects in the real setting by using markerless computer vision based tracking in addition to GPS localization. We further intend to include a more high-level game description language as well as graphical editing and orchestration tools to allow for an easier and faster setup of this kind of pervasive AR games as well as an easier adaptation to alternate locations (further cities).

\section{ACKNOWLEDGMENT}

The authors thank their colleagues at the Collaborative Virtual and Augmented Environments Department at Fraunhofer FIT for their comments and contributions. They further wish to thank their project partners of the IPCity project for their ideas, cooperation, and their support. IPCity (FP6-2004-IST-4-27571) is partially funded by the European Commission as part of the $6^{\text {th }}$ Framework.

\section{REFERENCES}

[1] Benyon, D. Smyth, M., O'Neill, S., McCall, R. and Carrol, F. The Place Probe: Exploring a Sense of Place in Real and Virtual Environments. Journal of Presence: Tele-operators and Virtual Environments. 15, 6, (2006) 668-687.

[2] Broll, W., Ohlenburg, J., Lindt, I., Herbst, I., and Braun, A.-K.:" Meeting Technology Challenges of Pervasive Augmented Reality Games". In Proc. of the ACM Netgames 2006 (Singapore, Oct. 3031, 2006). Also published in ACM Digital Library.

[3] Ohlenburg, J., Herbst, I., Lindt, I., Fröhlich, T., and Broll, W. 2004. The MORGAN Framework: Enabling Dynamic Multi-User AR and VR Projects. In Proceedings of the ACM Symposium on Virtual Reality Software and Technology (VRST 2004, Hong-Kong). 